\title{
Effectiveness of Tocilizumab with and without Dexamethasone in Patients with Severe COVID-19: A Retrospective Study
}

\author{
Dorota Zarębska-Michaluk, (iD) \\ Jerzy Jaroszewicz, ${ }^{2}$ Magdalena Rogalska, ${ }^{3}$ \\ Diana Martonik, ${ }^{3}$ Paweł Pabjan, (D) ${ }^{\prime}$ \\ Aleksandra Berkan-Kawińska, ${ }^{4}$ \\ Beata Bolewska, ${ }^{5}$ Barbara Oczko-Grzesik, ${ }^{2}$ \\ Dorota Kozielewicz, ${ }^{6}$ Magdalena Tudrujek- \\ Zdunek, ${ }^{7}$ Justyna Kowalska, ${ }^{8}$ \\ Anna Moniuszko-Malinowska, \\ Krzysztof Kłos, (D) ${ }^{10}$ Marta Rorat, ${ }^{11,12}$ \\ Piotr Leszczyński, ${ }^{13,14}$ Anna Piekarska, \\ Joanna Polańska, iD ${ }^{15}$ Robert Flisiak ${ }^{3}$ \\ 'Department of Infectious Diseases, Jan \\ Kochanowski University, Kielce, 25-369, Poland; \\ ${ }^{2}$ Department of Infectious Diseases and \\ Hepatology, Medical University of Silesia, \\ Katowice, 40-055, Poland; ${ }^{3}$ Department of \\ Infectious Diseases and Hepatology, Medical \\ University of Białystok, Białystok, I5-089, Poland; \\ ${ }^{4}$ Department of Infectious Diseases and \\ Hepatology, Medical University of Łódź, Łódź, 90- \\ 549, Poland; ${ }^{5}$ Department of Infectious Diseases, \\ University of Medical Sciences, Poznań, 6I-70I, \\ Poland; ${ }^{6}$ Department of Infectious Diseases and \\ Hepatology, Faculty of Medicine, Collegium \\ Medicum in Bydgoszcz, Nicolaus Copernicus \\ University, Toruń, 87-100, Poland; ${ }^{7}$ Department of \\ Infectious Diseases and Hepatology, Medical \\ University of Lublin, Lublin, 20-059, Poland; \\ ${ }^{8}$ Department of Adults' Infectious Diseases, \\ Medical University of Warsaw, Warsaw, 02-091, \\ Poland; 'Department of Infectious Diseases and \\ Neuroinfections, Medical University of Białystok, \\ Białystok, 15-089, Poland; ${ }^{10}$ Department of \\ Infectious Diseases and Allergology, Military \\ Institute of Medicine, Warsaw, 04-I4I, Poland; \\ "Department of Forensic Medicine, Wrocław \\ Medical University, Wrocław, 50-367, Poland; \\ ${ }^{12}$ First Infectious Diseases Ward, Gromkowski \\ Regional Specialist Hospital in Wrocław, Wrocław, \\ 5I-I49, Poland; ${ }^{13}$ Department of Rheumatology, \\ Rehabilitation and Internal Medicine, Poznan \\ University of Medical Sciences, Poznań, 6I-70I, \\ Poland; ${ }^{14}$ Department of Rheumatology and \\ Osteoporosis, Szpital im. J. Strusia w Poznaniu, \\ Poznań, 6I-285, Poland; ${ }^{15}$ Silesian University of \\ Technology, Gliwice, 44-100, Poland
}

Correspondence: Dorota ZarębskaMichaluk

Department of Infectious Diseases, Jan Kochanowski University in Kielce, UI.

Radiowa 7, Kielce, 25-317, Poland

Tel +48662441465

$\mathrm{Fax}+48$ 4I 3682262

Email dorotal010@tlen.pl
Purpose: The pathogenesis of coronavirus disease 2019 (COVID-19) is complicated, and in addition to antiviral therapy and combating coagulopathy, treatment should also include inhibition of the proinflammatory cytokines overproduction. The purpose of this study is to compare the effectiveness of tocilizumab (TCZ) and dexamethasone (DEX) administered alone or in combination in patients with severe COVID-19.

Patients and Methods: Patients were selected from the SARSTer database, containing 3330 individuals with COVID-19 treated between 1 March 2020 and 10 March 2021. The current study included adult patients with baseline oxygen saturation $\left(\mathrm{SpO}_{2}\right) \leq 90 \%$, requiring regular or non-invasive high-flow oxygen supplementation.

Results: Among included 460 patients, 59 were treated with TCZ, 125 with TCZ and DEX, 169 with DEX, and 107 did not receive TCZ nor DEX. The groups were balanced regarding demographics, coexisting diseases, baseline $\mathrm{SpO}_{2}$, and comedications with remdesivir or low-molecular-weight heparin. The death rate of $6.8 \%$ was significantly lower in patients receiving TCZ alone than each arm (19.6\%-23.1\%), particularly in patients with interleukin6 concentration exceeding $100 \mathrm{pg} / \mathrm{mL}$ ( $5 \%$ vs $22.9 \%-51.7 \%$, respectively). Analysis of clinical improvement demonstrated doubled, significantly higher rate after 21 and 28 days in patients treated with TCZ alone (60\% and $75 \%$, respectively) compared to DEX $(27.6 \%$ and $37.9 \%$, respectively). The need for mechanical ventilation was similar in all arms.

Conclusion: In patients with severe course of COVID-19, particularly those developing cytokine storm, administration of TCZ provides a significantly better effect than DEX regarding survival, clinical improvement, and hospital discharge rate. The combination of TCZ and DEX does not improve therapy effectiveness in patients with severe COVID-19 compared to the administration of TCZ alone.

Keywords: SARS-CoV-2, COVID-19, tocilizumab, dexamethasone, cytokine storm

\section{Introduction}

The 2019 coronavirus disease (COVID-19) outbreak posed a huge epidemiological, diagnostic and therapeutic challenge. The availability of vaccines has given hope of calming this global crisis but has not diminished the need for effective therapy. The search for effective treatment options focused on repurposing approved drugs with proven activity in other diseases. The main recommendations were remdesivir, lowmolecular-weight heparin, and dexamethasone. However, the pathogenesis of COVID-19 is complicated, and in addition to its antiviral activity and combating coagulopathy, therapy should also include inhibition of the proinflammatory 
cytokines overproduction. This so-called cytokine storm is responsible for multiorgan damage and is believed to be the leading cause of COVID-19-related deaths. The first recommended method of cytokine storm control was dexamethasone, which has some effect in advanced disease in patients requiring mechanical ventilation. ${ }^{1}$ However, to prevent the development of this stage of COVID-19, which is characterized by high mortality, it is necessary to reduce significantly elevated levels of proinflammatory cytokines, including interleukin (IL)- $6 .^{2}$ It has been shown that higher levels of IL-6 are significantly associated with increased mortality in patients. ${ }^{3}$ Therefore, tocilizumab (TCZ), an inhibitor of IL-6 receptors, was considered a possible therapeutic option. ${ }^{2-5}$ Although some preliminary studies have not confirmed the efficacy of TCZ, possibly due to the heterogeneity of the studied populations, the authors have not ruled out a possible benefit of IL- 6 receptor blockade in some patient populations. ${ }^{6,7}$ Finally, the most recent and largest study by Salama et al showed a survival benefit in patients treated with TCZ. In addition, it has recently been demonstrated that this regimen may be particularly effective in reducing mortality and the need for mechanical ventilation in patients with baseline IL-6 levels greater than $100 \mathrm{pg} / \mathrm{mL}$, especially if they require supplementation with oxygen. ${ }^{3,8}$

The purpose of this study is to compare the effectiveness of tocilizumab and dexamethasone administered alone or in combination in patients with severe COVID-19.

\section{Patients and Methods}

Patients included in the current analysis originated from the SARSTer national database, which included 3330 patients treated between 1 March 2020 and 10 March 2021 in 30 Polish centers. This ongoing project, supported by the Polish Association of Epidemiologists and Infectiologists (PTEiLChZ) and Medical Research Agency, is a national real-world experience study assessing treatment in patients with COVID-19. The study was conducted according to the guidelines of the Declaration of Helsinki, and approved by the Ethics Committee of Medical University of Białystok (29 October 2020, number APK.002.303.2020). Additionally, the local bioethics committees approved the experimental use of tocilizumab in patients with COVID-19 and written informed consent was obtained from each patient before the treatment initiation.

All the patients were diagnosed with COVID-19 based on positive results of the real-time reverse transcriptasepolymerase chain reaction (RT-PCR) from the nasopharyngeal swab specimen. The decision about the treatment regimen was taken entirely by the treating physician concerning current knowledge and recommendations of the PTEiLChZ. ${ }^{9,10}$ Throughout the analyzed period, tocilizumab and dexamethasone were recommended in patients with respiratory failure and cytokine storm in accordance with these national guidelines. It was conditionally possible to use dexamethasone in an earlier phase of disease related to viral replication as long as the patient was receiving remdesivir., ${ }^{9,10}$ The national COVID19 treatment guidelines, created by PTEiLChZ, were stable with respect to the administration of DEX and TCZ, and included these drugs in the first issue from March 2020 and the most recent one from April 2021.

The current study included 460 adult patients with baseline oxygen saturation $\left(\mathrm{SpO}_{2}\right) \leq 90 \%$, classified according to the ordinal scale as a hospitalized requiring regular oxygen supplementation (score 5) or on noninvasive ventilation with high-flow oxygen equipment (score 6). Patients with acute respiratory distress syndrome (ARDS) at baseline and those receiving monoclonal antibody directed against cytokine or viral receptors except tocilizumab were excluded. Among included 460 patients, 59 were treated with TCZ, 125 with TCZ and dexamethasone (DEX), 169 with DEX, and 107 did not receive TCZ nor DEX. Tocilizumab (RoActemra, Roche Pharma AG) was administered intravenously at $8 \mathrm{mg} / \mathrm{kg}$ (maximum dose: $800 \mathrm{mg}$ ) in a single dose (1-h infusion) after exclusion of severe bacterial and HBV infection, in patients with a neutrophil count $\geq 2 \times 10^{9} / 1$, a platelet count $\geq 50 \times 10^{3} / 1$ and alanine aminotransferase levels below 5 times the upper limit of normal. If no improvement was observed, the second dose was considered after 8-12 $\mathrm{h}$ according to the national recommendations. ${ }^{9,10}$ Data were entered retrospectively and submitted online by a web-based platform operated by Tiba sp. z o.o. Baseline data included age, gender, body mass index (BMI), coexisting conditions, other medication-related to COVID-19, $\mathrm{SpO}_{2}$, and laboratory measures of inflammation, including $\mathrm{C}$-reactive protein (CRP), procalcitonin, white blood cells (WBC), platelets, interleukin 6 (IL-6), and d-dimers. To differentiate patients regarding survival prognosis while on tocilizumab therapy, we applied a threshold of baseline serum IL-6 concentration of 100 $\mathrm{pg} / \mathrm{mL}$, which we established previously. ${ }^{3}$

The end-points of treatment effectiveness were death rate, need for mechanical ventilation, rate of discharge from the hospital on day 14,21 , or 28 , and rate of clinical 
improvement measured according to the ordinal scale. This scale is based on WHO recommendations modified to fit the specificity of the national health care system as applied previously. ${ }^{11,12}$ Clinical improvement was defined as at least a 2-point decrease from baseline to 14, 21, and 28 days of hospitalization. The ordinal scale was scored as follows: 1) unhospitalized, no activity restrictions; 2) unhospitalized, no activity restrictions and/or requiring oxygen supplementation at home; 3 ) hospitalized, does not require oxygen supplementation and does not require medical care; 4) hospitalized, requiring no oxygen supplementation, but requiring medical care; 5) hospitalized, requiring normal oxygen supplementation; 6) hospitalized, on non-invasive ventilation with high-flow oxygen equipment; 7) hospitalized, for invasive mechanical ventilation or extracorporeal membrane oxygenation (ECMO); and 8) death.

\section{Statistical Analysis}

The results were expressed as mean \pm standard deviation (SD) or $\mathrm{n}(\%)$. $\mathrm{P}$ values of $<0.05$ were considered to be statistically significant. The significance of difference was calculated by chi-square or Fisher's exact tests (as appropriate) for nominal variables and by Mann-Whitney $U$ and Kruskal-Wallis ANOVA for continuous and ordinal variables. Due to the variability in group size, the Fisher's p-values were accompanied by odds ratio (OR) as the sample size-independent effect size measures. Survival analyses were performed by Log rank (Mantel-ox) test supported by the Mantel-Haenszel hazard ratio (MH HR) and its 95\% confidence interval as the effect size measure. Survival analyses were depicted as Kaplan-Meier (KM) plot. Statistical analyses were performed by GraphPad Prism 5.0 (GraphPad Software, Inc., La Jolla, CA, USA).

\section{Results}

\section{Patient Characteristics}

Among 184 patients treated with TCZ alone or in combination with DEX, 95 (52\%) received a second dose of TCZ. As shown in Table 1, groups were balanced regarding gender, age, BMI, accompanying diseases, and comedications with remdesivir or low-molecular-weight heparin. There were also no statistically significant differences between particular groups regarding baseline $\mathrm{SpO}_{2}$ values, platelet counts, and D-dimers concentration. The median time between diagnosis and medication was 3 and 2 days, respectively, whereas median time between the symptoms onset and medication was 9 days for both regimens. The highest CRP and IL-6 were noticed in both arms treated with TCZ (Table 1).

\section{Treatment Effectiveness}

As shown in Table 2, the death rate of $6.8 \%$ was significantly lower in patients receiving TCZ alone than each arm, which demonstrated rates between $19.6 \%$ and $23.1 \%$. Further analysis performed by Log rank Mantel-Cox test and depicted as a Kaplan-Meier plot showed a significantly better survival

Table I Baseline Characteristics of Patients Included in the Study

\begin{tabular}{|c|c|c|c|c|c|}
\hline & $\mathbf{T C Z}$ & DEX & TCZ/DEX & No-TCZ/no-DEX & $\mathbf{P}$ \\
\hline & $N=59$ & $N=169$ & $N=125$ & $N=107$ & \\
\hline Females/males (n, \%) & $27 / 32,46 / 54$ & 65/104, 39/6I & $47 / 78,38 / 62$ & $41 / 66,38 / 62$ & 0.73 \\
\hline Age, years (mean, SD) & $63.4,12.5$ & $64.4,14.9$ & $64.4,14.9$ & $67.0,15.0$ & 0.24 \\
\hline BMI (mean, SD) & $29.7,5.2$ & $29.1,5.3$ & $29.6,5.2$ & $29.7,6.4$ & 0.79 \\
\hline Accompanying diseases (n, \%) & $50,84.7$ & $144.0,85.2$ & I0I, 80.8 & $91,85.0$ & 0.75 \\
\hline Remdesivir (n, \%) & $22,37.3$ & $71,42.0$ & $40,32.0$ & $42,39.3$ & 0.37 \\
\hline Heparin (n, \%) & $53,89.8$ & $143,84.6$ & $109,87.2$ & $83,77.6$ & 0.12 \\
\hline Convalescent plasma (n, \%) & $7,11.9$ & $40,23.7$ & $29,23.2$ & II, 10.3 & 0.01 \\
\hline Low-flow oxygen (n, \%) & $54,92.5$ & $153,90.5$ & $117,93.6$ & $100,93.5$ & 0.74 \\
\hline High-flow oxygen (n, \%) & $5,8.5$ & $16,9.5$ & $8,6.4$ & $7,6.5$ & 0.74 \\
\hline $\mathrm{SpO}_{2}, \%$ (mean, SD) & $85.4,6.0$ & $84.9,6.5$ & $84.3,5.8$ & $85.1,5.5$ & 0.34 \\
\hline CRP, mg/l (mean, SD) & $129.6,103.5$ & $104.9,72.4$ & 142.3, 79.4 & II $0.0,77.3$ & $<0.001$ \\
\hline Procalcitonin, $\mathrm{ng} / \mathrm{mL}$ (mean, SD) & $0.3,0.5$ & $0.5,2.1$ & $0.7,3.1$ & $0.6,2.0$ & 0.02 \\
\hline WBC, / $\mu \mathrm{l}$ (mean, SD) & 6108,2819 & 7792,3482 & $844 I, 4136$ & 7855,5835 & $<0.001$ \\
\hline Platelets, $\times 10^{3} / \mu \mid$ (mean, SD) & 207,86 & 228,101 & 223,85 & 224,113 & 0.52 \\
\hline IL-6, pg/mL (mean, SD) & 139,239 & 71,141 & 192,253 & 77,116 & $<0.001$ \\
\hline D-dimers, ng/mL (mean, SD) & 1360,2418 & $3823,14,745$ & 2884,7296 & $284 I, 6477$ & 0.21 \\
\hline
\end{tabular}


Table 2 Treatment Effectiveness Among All Patients Included in the Study

\begin{tabular}{|c|c|c|c|c|c|c|c|}
\hline & TCZ & DEX & $\begin{array}{l}\text { TCZI } \\
\text { DEX }\end{array}$ & $\begin{array}{l}\text { No-TCZ/no- } \\
\text { DEX }\end{array}$ & DEX vs TCZ & TCZ/DEX vs TCZ & $\begin{array}{l}\text { no-TCZ/no-DEX vs } \\
\text { TCZ }\end{array}$ \\
\hline & $\begin{array}{l}N=59 \\
n(\%)\end{array}$ & $\begin{array}{l}N=169 \\
n(\%)\end{array}$ & $\begin{array}{l}N=125 \\
n(\%)\end{array}$ & $\begin{array}{l}N=107 \\
n(\%)\end{array}$ & \multicolumn{3}{|l|}{ P, OR ( $\pm 95 \% \mathrm{Cl})$} \\
\hline Death & $4(6.8)$ & $39(23.1)$ & $28(22.4)$ & $21(19.6)$ & $0.006,0.24(0.08-0.71)$ & $0.01,0.25(0.09-0.76)$ & $0.04,0.30(0.10-0.91)$ \\
\hline Mechanical ventilation & $4(6.8)$ & $15(8.9)$ & $18(14.4)$ & $10(9.3)$ & $0.79,0.75(0.24-2.35)$ & $0.15,0.43(0.14-1.34)$ & $0.77,0.70(0.2 \mathrm{I}-2.36)$ \\
\hline Improvement on day 14 & $21(35.6)$ & $81(47.9)$ & $50(40.0)$ & $43(40.2)$ & $0.12,0.60(0.32-1.11)$ & $0.62,0.83(0.43-1.57)$ & $0.62,0.82(0.42-1.59)$ \\
\hline Improvement on day 21 & $34(57.6)$ & III (65.7) & $73(58.4)$ & $67(62.6)$ & $0.28,0.71(0.39-1.30)$ & $1.00,0.97(0.52-1.81)$ & $0.62,0.82(0.42-1.55)$ \\
\hline Improvement on day 28 & $44(74.6)$ & $122(72.2)$ & $87(69.6)$ & $75(70.1)$ & $0.86,1.13(0.58-2.22)$ & $0.60,1.28(0.63-2.58)$ & $0.59,1.25(0.6 \mathrm{I}-2.56)$ \\
\hline Discharged on day 14 & $16(27.1)$ & $76(45.0)$ & $46(36.8)$ & 37 (34.6) & $0.02,0.45(0.24-0.87)$ & $0.24,0.64(0.32-1.26)$ & $0.39,0.70(0.35-1.42)$ \\
\hline Discharged on day 21 & $30(50.8)$ & $110(65.1)$ & $73(58.4)$ & $64(59.8)$ & $0.06,0.55(0.30-1.01)$ & $0.35,0.74(0.40-1.37)$ & $0.32,0.69(0.37-1.32)$ \\
\hline Discharged on day 28 & $43(72.9)$ & $119(70.4)$ & $87(69.6)$ & $73(68.2)$ & $0.87,1.13(0.58-2.19)$ & $0.73,1.17(0.59-2.34)$ & $0.60,1.25(0.62-2.53)$ \\
\hline
\end{tabular}

rate in patients treated with TCZ than others (Figure 1). In patients with IL- $6 \geq 100 \mathrm{pg} / \mathrm{mL}$, treatment with DEX or noTCZ/no-DEX resulted in a significantly higher death rate (51.7\% and $45.5 \%$, respectively) compared to TCZ alone (5\%), (Table 3). In contrast, patients with IL-6 $<100 \mathrm{pg} / \mathrm{mL}$ death rate did not demonstrate significant differences between particular regimen arms including no-TCZ/noDEX (Table 4).

The need for mechanical ventilation was similar in all arms, but in patients with IL- $6 \geq 100 \mathrm{pg} / \mathrm{mL}$ treated with DEX, a significantly higher percentage was shown (31\%) compared to no such cases among receiving TCZ alone (Table 3). Analysis of clinical improvement rate carried out in this population demonstrated doubled, significantly higher clinical improvement rate after 21 and 28 days in patients treated with TCZ alone $(60 \%$ and $75 \%$, respectively) compared to DEX ( $27.6 \%$ and $37.9 \%$, respectively) (Table 3). There were no such differences regarding clinical improvement while analysis was carried out either in the whole population or in patients with IL- $6<100 \mathrm{pg} / \mathrm{mL}$

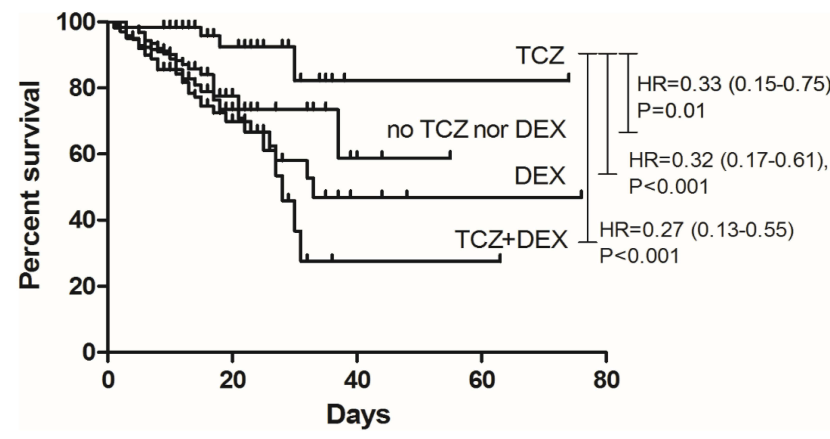

Figure I Kaplan-Meier plots of patients survival in particular arms by Log rank Mantel-Cox test supported by the Mantel-Haenszel hazard ratio (HR) and its $95 \%$ confidence interval.
(Tables 2 and 4). The rate of discharge from the hospital on day 14 was significantly higher in DEX- $(45 \%)$ than TCZ (27.1\%)-treated patients, but the differences faded away on days 21 and 28 (Table 2). A similar tendency was observed in a population of patients with IL-6 $<100 \mathrm{pg} / \mathrm{mL}$ (Table 4). In contrast, among patients with IL- $6 \geq 100 \mathrm{pg} / \mathrm{mL}$, these treated with TCZ demonstrated on day 28 a significantly higher discharge rate $(70 \%)$ compared to patients treated with DEX (34.5\%), (Table 3).

\section{Discussion}

Patients with COVID-19 who progress into cytokine storm are at risk of death due to hyperactivation and dysregulation of the immune response. Considering the pathogenesis of the systemic hyperinflammatory syndrome, the benefits of the immunomodulatory therapy at this stage of the disease were studied in the randomized clinical trials and observational RWE cohorts. ${ }^{1,3,4,6-8,13,14}$ Among drugs, the positive effect of which was assumed, corticosteroids and tocilizumab, were most often analyzed.

In the current real-world experience study, we evaluated and compared the impact of different immunomodulatory management in patients hospitalized with severe COVID-19 defined as baseline $\mathrm{SpO} 2 \leq 90 \%$ on air and requirement of the regular or non-invasive high-flow oxygen supplementation. With no differences between treatment arms in terms of demographics, comorbidities, and baseline severity status assessed by the rate of patients on regular and high-flow oxygen support, we found a significantly lower 28-day mortality rate among patients treated with TCZ as compared to those receiving DEX, combined therapy with TCZ/DEX and untreated individuals. The difference in death rate between TCZ and 
Table 3 Treatment Effectiveness Among Patients with IL-6 $2100 \mathrm{pg} / \mathrm{mL}$

\begin{tabular}{|c|c|c|c|c|c|c|c|}
\hline & TCZ & DEX & $\begin{array}{l}\text { TCZI } \\
\text { DEX }\end{array}$ & $\begin{array}{l}\text { No-TCZ } \\
\text { /no-DEX }\end{array}$ & DEX vs TCZ & TCZ/DEX vs TCZ & $\begin{array}{l}\text { No-TCZ/no-DEX vs } \\
\text { TCZ }\end{array}$ \\
\hline & $\begin{array}{l}N=20 \\
n(\%)\end{array}$ & $\begin{array}{l}N=29 \\
n(\%)\end{array}$ & $\begin{array}{l}N=70 \\
\text { n (\%) }\end{array}$ & $\begin{array}{l}N=22 \\
\text { n (\%) }\end{array}$ & \multicolumn{3}{|l|}{ P, OR ( $\pm 95 \% \mathrm{Cl})$} \\
\hline Death & I (5.0) & $15(5 \mid .7)$ & $16(22.9)$ & $10(45.5)$ & $<0.00 \mathrm{I}, 0.05(0.01-0.42)$ & $0.11,0.18(0.02-1.43)$ & $0.004,0.06(0.01-0.56)$ \\
\hline Mechanical ventilation & $0(0)$ & $9(31.0)$ & $10(14.3)$ & $5(22.7)$ & $0.007,0.05(0.01-0.97)$ & $0.1 \mathrm{I}, 0.14(0.0 \mathrm{I}-2.5 \mathrm{I})$ & $0.05,0.08(0.01-1.50)$ \\
\hline Improvement on day 14 & $6(30.0)$ & $3(10.3)$ & $25(35.7)$ & $3(13.6)$ & $0.13,3.7 \mid(0.80-17.2)$ & $0.79,0.77(0.26-2.26)$ & $0.27,2.7 \mid(0.58-12.8)$ \\
\hline Improvement on day 21 & $12(60.0)$ & $8(27.6)$ & $4 \mid(58.6)$ & $7(31.8)$ & $0.04,3.94(1.17-13.2)$ & $1.00,1.06(0.38-2.93)$ & $0.12,3.21(0.91-11.4)$ \\
\hline Improvement on day 28 & $15(75.0)$ & II (37.9) & $49(70.0)$ & $10(45.5)$ & $0.02,4.91(1.39-17.3)$ & $0.78,1.29(0.4 I-4.00)$ & $0.07,3.60(0.97-13.4)$ \\
\hline Discharged on day 14 & $4(20.0)$ & $3(10.3)$ & $24(34.3)$ & $2(9.1)$ & $0.42,2.17(0.43-10.9)$ & $0.28,0.48(0.14-1.60)$ & $0.40,2.50(0.40-15.4)$ \\
\hline Discharged on day 21 & $10(50.0)$ & $8(27.6)$ & $4 \mid(58.6)$ & $7(31.8)$ & $0.14,2.62(0.79-8.69)$ & $0.61,0.7 \mid(0.26-1.92)$ & $0.34,2.14(0.61-7.5 \mathrm{I})$ \\
\hline Discharged on day 28 & $14(70.0 \%)$ & $10(34.5)$ & $49(70.0)$ & $10(45.5)$ & $0.02,4.43(1.30-15.1)$ & $1.00,1.00(0.34-2.96)$ & $0.13,2.80(0.78-10.0)$ \\
\hline
\end{tabular}

Table 4 Treatment Effectiveness Among Patients with IL-6<100pg/mL

\begin{tabular}{|c|c|c|c|c|c|c|c|}
\hline & TCZ & DEX & $\begin{array}{l}\text { TCZI } \\
\text { DEX }\end{array}$ & $\begin{array}{l}\text { No-TCZ/no- } \\
\text { DEX }\end{array}$ & DEX vs TCZ & TCZ/DEX vs TCZ & $\begin{array}{l}\text { No-TCZ/no-DEX vs } \\
\text { TCZ }\end{array}$ \\
\hline & $\begin{array}{l}N=39 \\
n(\%)\end{array}$ & $\begin{array}{l}N=140 \\
n(\%)\end{array}$ & $\begin{array}{l}N=55 \\
n(\%)\end{array}$ & $\begin{array}{l}N=85 \\
n(\%)\end{array}$ & \multicolumn{3}{|l|}{ P, OR ( $\pm 95 \% \mathrm{CI})$} \\
\hline Death & $3(7.7)$ & $24(17.1)$ & $12(21.8)$ & II (12.9) & $0.21,0.40(0.11-1.4 I)$ & $0.09,0.30(0.08-1.14)$ & $0.54,0.56(0.15-2.14)$ \\
\hline Mechanical ventilation & $4(10.3)$ & $6(4.3)$ & $8(14.5)$ & $5(5.9)$ & $0.23,2.55(0.68-9.54)$ & $0.75,0.67(0.18-2.4 I)$ & $0.46,1.83(0.46-7.22)$ \\
\hline Improvement on day 14 & $15(38.5)$ & $78(55.7)$ & $25(45.5)$ & $40(47.1)$ & $0.07,0.50(0.24-1.03)$ & $0.53,0.75(0.32-1.73)$ & $0.44,0.70(0.32-1.52)$ \\
\hline Improvement on day 21 & $22(56.4)$ & $103(73.6)$ & $32(58.2)$ & $60(70.6)$ & $0.05,0.46(0.22-0.97)$ & $1.00,0.93(0.4 I-2.13)$ & $0.15,0.54(0.25-1.18)$ \\
\hline Improvement on day 28 & $29(74.4)$ & III (79.3) & $38(69.1)$ & $65(76.5)$ & $0.51,0.76(0.33-1.73)$ & $0.65,1.30(0.52-3.25)$ & $0.82,0.89(0.37-2.14)$ \\
\hline Discharged on day 14 & $12(30.8)$ & $73(52.1)$ & $22(40.0)$ & $35(41.2)$ & $0.02,0.41(0.19-0.87)$ & $0.39,0.67(0.28-1.59)$ & $0.32,0.63(0.28-1.42)$ \\
\hline Discharged on day 21 & $20(51.3)$ & $102(72.9)$ & $32(58.2)$ & $57(67.1)$ & $0.02,0.39(0.19-0.81)$ & $0.53,0.76(0.33-1.73)$ & $0.11,0.52(0.24-1.21)$ \\
\hline discharged on day 28 & $29(74.4)$ & $109(77.9)$ & $38(69.1)$ & $63(74.1)$ & $0.67,0.82(0.36-1.88)$ & $0.65,1.30(0.52-3.25)$ & I.00, I.0I (0.42-2.4I) \\
\hline
\end{tabular}

DEX treatment was even more remarkable when the comparison was conducted in a population with baseline IL$6 \geq 100 \mathrm{pg} / \mathrm{mL}$ corresponded to a hyperinflammatory state. Despite a small number of patients (20 and 29), the statistical significance of difference was documented, $5 \%$ vs $51.7 \%(\mathrm{p}<0.001)$.

Our findings on reducing the mortality rate with TCZ are in line with the results of the most recent analysis from a randomized trial RECOVERY. ${ }^{13}$ The improvement of survival, probability of discharge from hospital within 28 days, and reduction in the risk of progressing to require mechanical ventilation were reported among 2022 patients treated with TCZ compared to usual care. The beneficial effect of TCZ was documented in patients receiving both non-invasive and invasive oxygen support. Although the overall conclusions of the analysis are consistent with ours, the positive impact of TCZ was larger in patients who also received the systemic corticosteroids $(82 \%$ of the analyzed cohort), which is contrary to findings from the current study. However, it should be noted that according to baseline characteristics, the studied population differs from ours regarding oxygen requirement, 13\% was at baseline on the invasive mechanical ventilation, $41 \%$ received non-invasive high-flow support, whereas the majority of patients in our study received regular lowflow support, $92.5 \%$ and $93.6 \%$ in TCZ and TCZ/DEX arms, respectively. Moreover, the assessment of the eligibility to randomization was based on the CRP level without the measurement of the IL- 6 concentration, and these disparities could affect the results and explain the differences in the conclusions.

A significant proportion of patients on mechanical ventilation was also included in the COVACTA trial comparing TCZ and placebo in severe COVID-19 related pneumonia. ${ }^{4}$ No improvement of clinical status and the lower mortality rate was demonstrated for the use of TCZ in 294 patients compared to 144 individuals in the placebo group, but $19.4 \%$ and $28.5 \%$ of the participants also received corticosteroids, which makes the comparison with our results impossible. 
The EMPACTA trial studied a population more similar to ours, which consisted of hospitalized patients with COVID-19 pneumonia not receiving mechanical ventilation, of whom 249 were assigned to TCZ and 128 to placebo. ${ }^{8}$ Although the likelihood of progression to the composite outcome of mechanical ventilation or death was reduced in the TCZ arm, no improvement of the survival was documented. Noteworthy, in both TCZ and placebo groups, more than $80 \%$ received systemic corticosteroids, so detailed data on the effectiveness of TCZ and corticosteroids separately and in combination was no provided again. Also, the results from several randomized trials, that included 60-67 patients, assessing the use of TCZ did not support the findings on the significant reduction in mortality. ${ }^{6,15,16}$ The small numbers of included patients may reduce the statistical power to detect a treatment effect on the outcome of the disease, while the RECOVERY is the largest trial to date assessing the efficacy of interventions of COVID-19.

Another therapeutic option evaluated within this study in the large population of hospitalized COVID-19 patients with different levels of respiratory support was DEX, given in a dose of $6 \mathrm{mg}$ once daily. ${ }^{1}$ Significantly lower 28-day mortality rates were demonstrated in 2104 individuals treated with DEX compared to 4321 receiving the usual care, $22.9 \%$ vs $25.7 \%$, respectively. Interestingly, $8 \%$ of those assigned to the usual care arm were treated with DEX as part of their clinical care. According to the baseline characteristics, the studied cohort in both treatment arms presented more severe clinical presentation compared to our analysis with $15 \%, 16 \%$ of the patients on the mechanical ventilation and $61 \%, 60 \%$ on the noninvasive oxygen supplementation in DEX and usual care group, respectively. Since the greater impact on the death rate and probability of discharge was reported for the patients on the invasive mechanical ventilation, excluded from our analysis, it could be a possible explanation for the differences in the obtained results. The meta-analysis performed to estimate the association between the administration of corticosteroids, including DEX, and the mortality rate in critically ill patients with COVID-19 confirmed this population as the most beneficial from DEX treatment regardless of the dose. ${ }^{17}$ On the other hand, no effect of low dose DEX on the mortality rate was documented in the large real-world cohort of 1311 Italian patients with baseline $\mathrm{SpO} 2<90 \%$ not receiving invasive mechanical ventilation, and these results are consistent with ours. ${ }^{18}$
The current analysis demonstrated no benefit from combining TCZ with DEX. No randomized clinical trial has evaluated the combined therapy with tocilizumab and corticosteroids; only some RWE data, mainly with the use of methylprednisolone (MPS), are available with inconclusive results. The single-center retrospective study conducted among 255 Spanish patients with severe COVID-19 pneumonia, of whom 21 were assigned to TCZ, 38 to MPS, 78 were treated with both agents, and 118 did not receive immunomodulators, documented the greatest reduction of in-hospital mortality for the combination of corticosteroids and TCZ, with death rates $33.3 \%, 44.7 \%, 29.5 \%$, and $58.5 \%$, respectively. ${ }^{19}$ Similar results were achieved in the Italian single-center observational study performed by Mikulska et al. ${ }^{20}$ Among 196 non-intubated individuals with severe pneumonia included in the analysis, the lowest mortality rate of $8.9 \%$ was demonstrated in patients treated with combined therapy, whereas rates reported in those treated with TCZ and MPS were $13.8 \%$ and $28.9 \%$, respectively. The same corticosteroid formulation was also used in the SAM-COVID study, which aimed to compare treatment with TCZ, MPS, or combined therapy performed by Rodriguez-Bano et al in patients with the hyperinflammatory state. ${ }^{21}$ This RWE project included 434 patients receiving treatment and 344 untreated individuals. Similarly to our study, patients on mechanical ventilation at the admission were excluded from the analysis, and the majority of participants in all therapeutic options required low-flow oxygen supplementation, $92.8 \%, 95.7 \%, 97.4 \%, 91.3 \%$, and $96.4 \%$ in TCZ, corticosteroids applied in intermediate-high or pulse dose, combined treatment and untreated population, respectively. The main endpoint was mechanical ventilation or death, depending on what happened first, achieved by $11.4 \%, 23.1 \%, 15.4 \%, 25.6 \%$, and $21.1 \%$ in the abovementioned study arms. Based on the results of this study, very similar in design to ours, the authors concluded that TCZ might be useful in patients with a hyperinflammatory state and therefore should be prioritized in the research, and this summary supported our observations.

Comparing the results of our analysis to other studies, the critical issue that requires further research is identifying the patients likely to benefit from TCZ and corticosteroids and the timing to implement such therapies to optimize the outcome.

The current study has several limitations that we are aware of, and some of them are related to its observational and retrospective nature. The choice of the immunomodulatory treatment was made by treating physicians based on 
medical knowledge and national recommendations without randomization. We did not evaluate the impact of the timing of drug application on the outcome of the disease. Focusing on the efficacy of the assigned medications, we did not analyze the safety data for this report. And finally, the electronic data capture could result in possible data entry errors. Since our study is observational, we must consider the possibility of an immortal time bias. However, in all groups, observation was started at the admission to the hospital, analyzed drugs were usually introduced during the first days of hospitalization and median time between symptom onset and drug administration was exactly the same for tocilizumab and dexamethasone.

The major strength of the current analysis is the collection of data from a real-world experience study, which is a representative of routine clinical practice, but also the availability of baseline serum IL-6 in all studied patients. However, the final analysis was conducted on a relatively homogeneous population of severely but not critically ill patients including those entering the cytokine storm. Effective pharmacotherapy at this stage of the disease can prevent mechanical ventilation, which significantly affects the chances of survival.

\section{Conclusions}

In patients with severe course of COVID-19, particularly those developing cytokine storm, administration of tocilizumab provides a significantly better effect than dexamethasone regarding survival, clinical improvement, and hospital discharge rate. The combination of tocilizumab and dexamethasone does not improve therapy effectiveness in these patients compared to the administration of tocilizumab alone.

\section{Data Sharing Statement}

The original anonymous dataset is available on request from the corresponding author at dorota1010@tlen.pl.

\section{Funding}

Medical Research Agency, grant number 2020/ABM/ COVID19/PTEILCHZ and Polish Association of Epidemiologists and Infectiologists.

\section{Disclosure}

Dr Dorota Zarębska-Michaluk reports grants from Agencja Badań Medycznych, during the conduct of the study; personal fees from Gilead, personal fees from AbbVie, outside the submitted work. Dr Jerzy Jaroszewicz reports grants, non-financial support from Gilead, personal fees, non-financial support from Roche, personal fees from
Bausch Health, outside the submitted work. Professor Robert Flisiak reports grants, personal fees from Roche, during the conduct of the study; grants, personal fees from Gilead, grants, personal fees from AbbVie, grants, personal fees from MSD, personal fees from Adamed, personal fees from Pfizer, outside the submitted work. The authors declare no other competing interests in this work.

\section{References}

1. Horby P, Lim WS, Emberson JR, et al.; RECOVERY Collaborative Group. Dexamethasone in hospitalized patients with Covid-19. N Engl J Med. 2021;384(8):693-704. doi:10.1056/NEJMoa2021436

2. Tomasiewicz K, Piekarska A, Stempkowska-Rejek J, et al. Tocilizumab for patients with severe COVID-19: a retrospective, multi-center study. Expert Rev Anti Infect Ther. 2021;19(1):93-100. doi:10.1080/14787210.2020.1800453

3. Flisiak R, Jaroszewicz J, Rogalska M, et al. Tocilizumab improves the prognosis of COVID-19 in patients with high IL-6. J Clin Med. 2021;10(8):1583. doi:10.3390/jcm10081583

4. Rosas IO, Bräu N, Waters $\mathrm{M}$, et al. Tocilizumab in hospitalized patients with severe Covid-19 pneumonia. $N$ Engl J Med. 2021;384 (16):1503-1516. doi:10.1056/NEJMoa2028700

5. Liu B, Li M, Zhou Z, Guan X, Xiang Y. Can we use interleukin-6 (IL-6) blockade for coronavirus disease 2019 (COVID-19)-induced cytokine release syndrome (CRS)? J Autoimmun. 2020;111:102452. doi:10.1016/j.jaut.2020.102452

6. Hermine O, Mariette X, Tharaux PL, Resche-Rigon M, Porcher R, Ravaud P, CORIMUNO-19 Collaborative Group. Effect of tocilizumab vs usual care in adults hospitalized with COVID-19 and moderate or severe pneumonia: a randomized clinical trial. JAMA Intern Med. 2021;181(1):32-40. doi:10.1001/jamainternmed.2020.6820

7. Stone JH, Frigault MJ, Serling-Boyd NJ, et al. BACC Bay Tocilizumab Trial Investigators. Efficacy of tocilizumab in patients hospitalized with Covid-19. N Engl J Med. 2020;383(24):2333-2344. doi:10.1056/NEJMoa2028836

8. Salama C, Han J, Yau L, et al. Tocilizumab in patients hospitalized with Covid-19 pneumonia. N Engl J Med. 2021;384(1):20-30. doi:10.1056/NEJMoa2030340

9. Flisiak R, Horban A, Jaroszewicz J, et al. Management of SARS-CoV-2 infection: recommendations of the Polish Association of Epidemiologists and Infectiologists as of March 31, 2020. Pol Arch Intern Med. 2020;130(4):352-357. doi:10.20452/pamw.15270

10. Flisiak R, Horban A, Jaroszewicz J, et al. Management of SARS-CoV- infection: recommendations of the Polish Association of Epidemiologists and Infectiologists as of April 26, 2021. Pol Arch Intern Med. 2021. doi:10.20452/pamw.15979

11. Flisiak R, Zarębska-Michaluk D, Berkan-Kawińska A, et al. Remdesivir-based therapy improved the recovery of patients with COVID-19 in the multicenter, real-world SARSTer study. Pol Arch Intern Med. 2021;131(1):103-110. doi:10.20452/pamw.15735

12. Moniuszko-Malinowska A, Czupryna P, Zarębska-Michaluk D, et al. Convalescent plasma transfusion for the treatment of COVID-19experience from Poland: a multicenter study. J Clin Med. 2020;10 (1):28. doi:10.3390/jcm10010028

13. RECOVERY Collaborative Group. Tocilizumab in patients admitted to hospital with COVID-19 (RECOVERY): a randomized, controlled, open-label, platform trial. Lancet. 2021;397(10285):1637-1645. doi:10.1016/S0140-6736(21)00676-0

14. Guaraldi G, Meschiari M, Cozzi-Lepri A, et al. Tocilizumab in patients with severe COVID-19: a retrospective cohort study. Lancet Rheumatol. 2020;2(8):e474-e484. doi:10.1016/S2665-9913(20)30173-9 
15. Veiga VC, Prats JAGG, Farias DLC, et al. Coalition covid-19 Brazil VI Investigators. Effect of tocilizumab on clinical outcomes at 15 days in patients with severe or critical coronavirus disease 2019: randomised controlled trial. BMJ. 2021;372:n84. doi:10.1136/bmj.n84

16. Salvarani C, Dolci G, Massari M, et al; RCT-TCZ-COVID-19 Study Group. Effect of tocilizumab vs standard care on clinical worsening in patients hospitalized with COVID-19 pneumonia: a randomized clinical trial. JAMA Intern Med. 2021;181(1):24-31. doi:10.1001/ jamainternmed.2020.6615

17. Sterne JAC, Murthy S, Diaz JV, et al.; WHO Rapid Evidence Appraisal for COVID-19 Therapies (REACT) Working Group. Association between administration of systemic corticosteroids and mortality among critically ill patients with COVID-19: a meta-analysis. JAMA. 2020;324(13):1330-1341. doi:10.1001/jama.2020.17023

18. Fusina F, Albani F, Granato E, et al. Effect of Corticosteroids on mortality in hospitalized COVID-19 patients not receiving invasive mechanical ventilation. Clin Pharmacol Ther. 2021;109(6):1660-1667. doi:10.1002/ cpt.2245
19. Van den Eynde E, Gasch O, Oliva JC, et al. Corticosteroids and tocilizumab reduce in-hospital mortality in severe COVID-19 pneumonia: a retrospective study in a Spanish hospital. Infect Dis. 2021;53(4):291-302. doi:10.1080/23744235.2021.1884286

20. Mikulska M, Nicolini LA, Signori A, et al. Tocilizumab and steroid treatment in patients with COVID-19 pneumonia. PLoS One. 2020;15 (8):e0237831. doi:10.1371/journal.pone.0237831

21. Rodríguez-Baño J, Pachón J, Carratalà J, et al.; SAM-COVID Study Group. Treatment with tocilizumab or corticosteroids for COVID-19 patients with hyperinflammatory state: a multicentre cohort study (SAM-COVID-19). Clin Microbiol Infect. 2021;27(2):244-252. doi:10.1016/j.cmi.2020.08.010
Journal of Inflammation Research

\section{Publish your work in this journal}

The Journal of Inflammation Research is an international, peerreviewed open-access journal that welcomes laboratory and clinical findings on the molecular basis, cell biology and pharmacology of inflammation including original research, reviews, symposium reports, hypothesis formation and commentaries on: acute/chronic inflammation; mediators of inflammation; cellular processes; molecular
Dovepress

mechanisms; pharmacology and novel anti-inflammatory drugs; clinical conditions involving inflammation. The manuscript management system is completely online and includes a very quick and fair peerreview system. Visit http://www.dovepress.com/testimonials.php to read real quotes from published authors. 